\title{
ON THE EXISTENCE AND SENSITIVITY ANALYSIS OF OPTIMAL CAPITAL ACCUMULATION PATHS IN CONTINUOUS TIME, INFINITE HORIZON MODELS
}

\author{
ELIAS FLYTZANIS ${ }^{1}$ and NIKOLAOS S. PAPAGEORGIOU ${ }^{2}$
}

(Received 8 October 1987; revised 10 May 1988)

\begin{abstract}
In this paper we consider an infinite horizon, continuous time model of economic growth. We prove two theorems; one on the existence of optimal paths of capital accumulation and the other on the dependence of the set of optimal paths on the initial capital stock (sensitivity analysis). In the existence result the underlying technology set is nonconvex and only its "investment" slices are convex. The proof is direct, without any use of necessary conditions. In the sensitivity analysis, the technology set is convex and so we have that the value function is concave. Then having that, we show that the set of optimal paths is an upper semicontinuous multifunction of the initial capital stock.
\end{abstract}

\section{Introduction}

In this paper, we consider a problem of capital accumulation with infinite horizon and we prove the existence of strongly optimal paths and determine their dependence on the initial capital stock.

Mathematical economists seem to have been the first to include systematically an infinite time horizon in their modelling of dynamical economic systems. Quoting Arrow and Kurz [1], we can say the following concerning the infinite time span: "The infinite horizon is an idealisation of the fundamental point that the consequences of investment are very long lived; any short horizon requires some methods of evaluating end-of-period capital stocks, and the only proper evaluation is their value in use in the subsequent future".

\footnotetext{
${ }^{1}$ Athens School of Economics \& Business Science, 76, Patission St., Athens 104-34, Greece.

${ }^{2}$ University of California, Department of Mathematics, Davis, California 95616, U.S.A.

(C) Copyright Australian Mathematical Society 1989, Serial-fee code 0334-2700/89
} 
The first to consider dynamical economic systems observed on an unbounded time interval, was Ramsey [14]. In his seminal work, Ramsey was able to derive optimality conditions for his economic model, which allowed for positive discount.

More sophisticated models were then studied by Mirrelees [12], Haurie [9] and Cass and Shell [6].

The model considered here is a general continuous time model of economic growth with infinite horizon and changing technology, similar to the model of Cass and Shell [6].

We establish the existence of optimal capital accumulation paths, without consideration of the necessary conditions for infinite horizon problems, existing in the literature (see for example Benveniste and Sheinkman [4] and Haurie [9]). Instead we use a direct approach that follows the fundamental works of Cesari [7] and Rockafellar [15].

Our existence theorem extends previous ones obtained by Gaines and Peterson [8] and Takekuma [17]. In both papers, the assumptions on the data of the problem are more restrictive and the mathematical techniques used are different. In [8], Gaines and Peterson use ideas from degree theory while Takekuma [17] uses some nice features of the Mackey topology on the space $L_{n}^{\infty}$ of essentially bounded functions on $R_{+}=[0, \infty)$ with values in $R^{n}$, i.e. $L_{n}^{\infty}=\left\{f: R_{+} \rightarrow\right.$ $R^{n}$ measurable such that $\left.\|f\|_{\infty}<\infty\right\}$, where $\|f\|_{\infty}=\inf \left\{M \in R_{+}: \lambda(t \in\right.$ $\left.\left.R_{+}:\|f(t)\| \geq M\right)=0\right\}$ (here $\lambda(\cdot)$ denotes the Lebesgue measure on $R_{+}$).

Here using techniques from the theory of multifunctions, as well as a recent powerful semicontinuity result of Balder [3], we are able to avoid all the extra restrictive hypotheses that the above two works have, and establish the existence of optimal paths, through a more direct and shorter procedure.

Our second result examines the relation between the optimal path and the initial capital stock and extends Theorem 4.2 of Takekuma [17]. This kind of analysis on the relation between the optimal path and some parameter(s) of the problem, is usually called "Sensitivity Analysis" and was initiated by the work of Brock [5].

Note that in both [8] and [17], the underlying technology set was assumed to be constant, while in this work we allow it to be time dependent. The first study that considered an optimal economic growth model with time changing technology, was that by Mirrelees [12].

\section{Preliminaries}

Our state space will be $R^{n}$ and the planning horizon will be $R_{+}=[0, \infty)$. For any elements $x, z \in R^{n}$, their inner product will be denoted by $\langle x, z\rangle$. The Euclidean norm of any $x \in R^{n}$ is denoted by $\|x\|$ i.e. $\|x\|=\langle x, x\rangle^{1 / 2}$. 
Throughout this paper we shall be using the following notations. Let $m \geq$ 1: $P_{f(c)}\left(R^{m}\right)=\left\{A \subseteq R^{m}:\right.$ nonempty, closed, (convex) $\}$ and $P_{k(c)}\left(R^{m}\right)=\{A \subseteq$ $R^{m}$ : nonempty, compact, (convex) $\}$.

Let $X, Y$ be two Hausdorff topological spaces. A multifunction (correspondence) $F(\cdot)$ from $X$ into $Y$, is a set-valued function of $X$ into $Y$ s.t. $F(x) \neq \varnothing$ for all $x \in X$. We say that $F(\cdot)$ is upper semicontinuous (u.s.c.), if for all $C \subset Y$ closed, the set $F^{-}(C)=\{x \in X: F(x) \cap C \neq \varnothing\}$ is closed in $X$.

In the sequel, all measure theoretic concepts that we will use (such as "measurable", "integrable" and "almost everywhere", abbreviated by a.e.), are defined in the sense of Lebesgue (see Ash [2]).

Let $f: R_{+} \rightarrow R^{n}$ be a measurable function. We define the following norm of $f(\cdot):\|f\|_{1}=\int_{0}^{\infty}\|f(s)\| d s$.

Also we say that two measurable functions $f_{1}, f_{2}: R_{+} \rightarrow R^{n}$ are equivalent, if and only if $f_{1}(t)=f_{2}(t)$ a.e. The space of all equivalence classes of measurable functions $f: R_{+} \rightarrow R^{n}$ such that $\|f\|_{1}<\infty$ is denoted by $L^{1}\left(R_{+}, R^{n}\right)=L_{n}^{1}\left(R_{+}\right)$. It is well known that $L_{n}^{1}$ with the $\|\cdot\|_{1}$-norm is a Banach space (see Ash [2]).

Similarly, on a measurable function $g: R_{+} \rightarrow R^{n}$ we can define another norm by $\|g\|_{\infty}=\inf \left\{M>0: \lambda\left(t \in R_{+}:\|g(t)\|>M\right)=0\right\}$.

Here $\lambda(\cdot)$ denotes the Lebesgue measure on $R_{+}$. The space of all equivalence classes of measurable functions $g: R_{+} \rightarrow R^{n}$ such that $\|g\|_{\infty}<\infty$ is denoted by $L^{\infty}\left(R_{+}, R^{n}\right)=L_{n}^{\infty}\left(R_{+}\right)$. This space with the $\|\cdot\|_{\infty}$-norm is a Banach space. Recall (see Ash [2]), that $\left\{f_{n}\right\}_{n \geq 1} \subseteq L_{n}^{1}\left(R_{+}\right)$is said to converge weakly to $f(\cdot) \in L_{n}^{1}\left(R_{+}\right)$(denoted by $f_{n} \stackrel{w}{\rightarrow} f$ in $L_{n}^{1}\left(R_{+}\right)$), if for all $g \in L_{n}^{\infty}\left(R_{+}\right)$, $\int_{0}^{\infty}\left\langle f_{n}(s), g(s)\right\rangle d s \rightarrow \int_{0}^{\infty}\langle f(s), g(s)\rangle d s$ as $n \rightarrow \infty$. The weak topology is weaker than the norm (strong) topology on $L_{n}^{1}\left(R_{+}\right)$. When on $L_{n}^{1}\left(R_{+}\right)$we consider the weak topology, we write $L_{n}^{1}\left(R_{+}\right)_{w}$.

Also by $C_{n}\left(R_{+}\right)$we denote the space of continuous functions from $R_{+}$into $R^{n}$. This is not a Banach space, since $R_{+}$is not compact. On $C_{n}\left(R_{+}\right)$we consider the topology of uniform convergence on compact subsets of $R_{+}$(also known to mathematicians as "compact-open topology"). So $\left\{f_{n}\right\}_{n \geq 1} \subseteq C_{n}\left(R_{+}\right)$converges to $f \in C_{n}\left(R_{+}\right)$in this topology if and only if $f_{n}(t) \rightarrow f(t)$ uniformly on compact subsets of $R_{+}$, that is, if $b \in R_{+}$and for any function $g: R_{+} \rightarrow R^{n},\left.g\right|_{b}(\cdot)$ is the restriction of $g(\cdot)$ on $[0, b]$, then $\left\|\left.f_{n}\right|_{b}-\left.f\right|_{b}\right\|_{\infty} \rightarrow 0$ as $n \rightarrow \infty$ for all $b \geq 0$ (recall that a continuous function is measurable and bounded on a compact set).

A subset $K \subseteq L_{n}^{1}\left(R_{+}\right)$is said to be "uniformly integrable" if $\lim _{\lambda(A) \rightarrow 0} \int_{A}\|f(t)\| d t=0$ uniformly in $f \in K$. From the Dunford-Pettis criterion (see for example [2] and [7]), we know that this notion is closely related to weak sequential compactness in $L_{n}^{1}\left(R_{+}\right)$. Namely the weak closure of $K$ is weakly sequentially compact if and only if $K$ is uniformly integrable. Note that 
if for every $f \in K,\|f(t)\| \leq \varphi(t)$ a.e. with $\varphi(\cdot) \in L_{+}^{1}$, then clearly $K$ is uniformly integrable.

A function $x: R_{+} \rightarrow R^{n}$ is said to be absolutely continuous, if given $\varepsilon>0$, there exists $\delta(\varepsilon)>0$ such that $\sum_{k=1}^{n}\left\|x\left(t_{k}^{\prime}\right)-x\left(t_{k}\right)\right\|<\varepsilon$ for all finite systems of nonoverlapping intervals $\left(t_{k}, t_{k}^{\prime}\right), k=1, \ldots, n$ with $\sum_{k=1}^{n}\left(t_{k}^{\prime}-t_{k}\right)<\delta$. A classical theorem of Lebesgue says that in this case $\dot{x}(\cdot)$ exists almost everywhere, for every $b>0, \dot{x}(\cdot) \in L_{n}^{\mathbf{1}}([0, b])$ (such a function is called "locally integrable" i.e. $\left.\dot{x}(\cdot) \in L_{\text {loc }}^{1}\left(R_{+}, R^{n}\right)\right)$ and for every $t \in T, x(t)=x(0)+\int_{0}^{t} \dot{x}(s) d s$.

Now that we have introduced the basic mathematical notions that we will need in the sequel, we can proceed to the description of the model. We assume that there are $n$ kinds of capital goods. So the state space in our model will be $R^{n}$. The production technology is time varying (a more natural assumption than the one made in [8] and [17], where the technology set was constant for the whole infinite time horizon). So we can model it as a multifunction $F(\cdot)$, from the planning horizon into the nonempty subsets of $R^{n} \times R^{n}$. If $(x, z) \in$ $F(t) \subseteq R^{n} \times R^{n}$, then this means that if at time instant $t$ the available capital stock is $x$, then level $z$ of investment is feasible. So we see that the technology constraint multifunction consists of all pairs of capital stocks and investment levels that are feasible at each time instant $t \in R_{+}$. Let $F_{1}(0)=\operatorname{proj}_{1} F(0)$ be the projection on the first factor of the technology set at time $t=0$, that is, $F_{1}(0)=\left\{x \in R^{n}:(x, z) \in F(0)\right.$ for some $\left.z \in R^{n}\right\}$. Roughly speaking, $F_{1}(0)$ consists of all possible initial capital stocks. An absolutely continuous function $x: R_{+} \rightarrow R^{n}$ is a path of capital accumulation and $\dot{x}(t)$ represents the level of investment at each time instant. If $x_{0} \in F_{1}(0)$, then a capital accumulation path is called "feasible and starting at $x_{0}$ " if and only if $(x(t), \dot{x}(t)) \in F(t)$ a.e. and $x(0)=x_{0}$.

We are also given a function $u: R_{+} \times R^{n} \times R^{n} \rightarrow R$, which at each point $(t, x, z) \in R_{+} \times R^{n} \times R^{n}$ gives us the maximum utility level that the economy can achieve at time $t$ when capital stock is $x$ and investment level is $y$. While in both [8] and [17], it is assumed that the utility function is jointly continuous in all three variables, here we will allow it to be measurable in $t$ and upper semicontinuous in $(x, z)$. Our hypotheses are more easily verifiable in concrete problems and broaden significantly the applicability of our model. Some growth hypotheses that we will eventually impose on both $F(\cdot)$ and $u(\cdot, \cdot, \cdot)$, are considerably less restrictive than the corresponding hypotheses in [8] and [17].

Our goal is to find a feasible path of capital accumulation, starting from a capital stock $x_{0} \in F_{1}(0)$, that minimises the time-aggregate utility $\int_{0}^{\infty} u(t, x(t), \dot{x}(t)) d t$. Such a path will be called "optimal". In mathematical terms we must solve the following variational problem:

$$
\int_{0}^{\infty} u(t, x(t), \dot{x}(t)) d t \rightarrow \sup
$$




$$
\begin{aligned}
\text { subject to : } & (x(t), \dot{x}(t)) \in F(t) \text { a.e., } \\
& \left.x(t) \geq 0 \text { (i.e. } x(t) \in R_{+}^{n}\right), \quad x(0)=x_{0} \in F_{1}(0) .
\end{aligned}
$$

\section{Existence of optimal paths}

In this section we shall establish the existence of optimal capital accumulation paths starting from a capital stock $x_{0} \in F_{1}(0)$. For this we will need the following hypotheses concerning the data of our variational problem $\left(^{*}\right)$.

$\underline{H(u)}: u: R_{+} \times R^{n} \times R^{n} \rightarrow R$ is a function s.t.

(1) $(t, x, z) \rightarrow u(t, x, z)$ is measurable,

(2) $(x, z) \rightarrow u(t, x, z)$ is u.s.c.,

(3) $z \rightarrow u(t, x, z)$ is concave,

(4) For every $x_{n} \rightarrow x$ in $C_{n}\left(R_{+}\right)$and $z_{n} \stackrel{w}{\rightarrow} z$ in $L_{n}^{1}\left(R_{+}\right)$, we have that $\left\{u^{+}\left(\cdot, x_{n}(\cdot), \dot{x}_{n}(\cdot)\right)\right\}_{n \geq 1}$ is uniformly integrable.

Recall that if $f: R_{+} \rightarrow R$ is measurable, $f^{+}=\max (f, 0)$ (i.e. $f^{+}$is the positive part of $f(\cdot))$. Hypotheses $H(u)(1)$ and (2) improve the standard assumption in the literature (see [8] and [17]), where $u(\cdot, \cdot, \cdot)$ is jointly continuous in all three variables. Hypothesis $H(u)(3)$ is a standard property of utility functions. Finally hypothesis $H(u)(4)$ has to do with the fact that our planning horizon is infinite.

$\underline{H(F)}: F: R_{+} \rightarrow P_{k}\left(R^{n} \times R^{n}\right)$ is a multifunction s.t.

(1) $F(\cdot)$ is u.s.c.

(2) there exists $N(\cdot) \in L^{\infty}\left(R_{+}\right)$s.t. for any technological process $(x(t), z(t))$ $\in F(t)$ a.e. with $\|x(t)\| \leq N(t)$ on $A \subseteq R_{+}$, we have $\langle x(t), z(t)\rangle \leq 0$ on A.

(3) for every $(t, x) \in R_{+} \times R^{n}, G(t, x)=\left\{z \in R^{n}:(x, z) \in F(t)\right\}$ is convex or empty and when it is nonempty we have $|G(t, x)|=\sup \{\|z\|: z \in$ $G(t, x)\} \leq \varphi(t,\|x\|)$ a.e. with $(t, r) \rightarrow \varphi(t, r)$ measurable, nondecreasing in $r$ and for any $b(\cdot) \in L_{+}^{\infty} \varphi(\cdot, b(\cdot)) \in L_{+}^{1}$.

The thing that we must emphasise about this set of hypotheses is the nonconvexity of the technology set. We only require the cross sections $G(t, x)$ to be convex (hypothesis $H(F)(3)$ ), allowing this way for increasing returns in production, which according to Cass and Shell [6] is an important case of economic dynamics. The growth condition on $|G(t, x)|$ simply says that the level of investment is limited by the availability of capital. Finally hypothesis $H(F)(2)$ says that when capital exists in sufficiently large quantities, the loss due to depreciation exceeds production.

Also in order for our variational problem $\left(^{*}\right)$ to have content, we make the following hypothesis. 
$H_{\alpha}\left(x_{0}\right)$ : There exists a feasible path $z(\cdot)$ starting at $x_{0}$ s.t. $\int_{0}^{\infty} u(t, z(t), \dot{z}(t)) d t$ is finite.

We have the following existence result for the capital accumulation problem $(*)$.

THEOREM 1. If hypotheses $H(u), H(F)$ and $H_{\alpha}\left(x_{0}\right)$ hold, then problem $\left({ }^{*}\right)$ admits an optimal capital accumulation path.

PROOF. Let $F_{1}(t)=\operatorname{proj}_{1} F(t)$, where $\operatorname{proj}_{1}(\cdot)$ denotes the projection on the first factor of $F(t) \subseteq R^{n} \times R^{n}$. Since by hypothesis $H(F)(1) F(\cdot)$ is u.s.c., Theorem 7.3.11 of Klein and Thompson [10] tells us that $F_{1}(\cdot)$ is u.s.c. Let $G r F_{1}$ denote the graph of $F_{1}(\cdot)$ i.e. $G r F_{1}=\left\{(t, x) \in R_{+} \times R^{n}: x \in F_{1}(t)\right\}$. Since $F_{1}(\cdot)$ is u.s.c., we know that $G r F_{1}$ is closed in $R_{+} \times R^{n}$.

Let $G: G r F_{1} \rightarrow P_{k}\left(R^{n}\right)$ be defined by $G(t, x)=\left\{z \in R^{n}:(x, z) \in F(t)\right\}$.

From hypothesis $H(F)(3)$, we know that $G(\cdot, \cdot)$ has convex values (i.e. for every $\left.(t, x) \in R_{+} \times R^{n}, G(t, x) \in P_{k c}\left(R^{n}\right)\right)$. Our claim is that $G(\cdot, \cdot)$ is an u.s.c. multifunction on $G r F_{1}$. So let $C \subset R^{n}$ be nonempty, closed. We need to show that $G^{-}(C)=\left\{(t, x) \in G r F_{1}: G(t, x) \cap C \neq \varnothing\right\}$ is closed. To this end, let $\left\{\left(t_{n}, x_{n}\right)\right\} \subseteq G^{-}(C)$ and assume that $\left(t_{n}, x_{n}\right) \rightarrow(t, x)$ in $G r F_{1}$. Let $z_{n} \in$ $G\left(t_{n}, x_{n}\right) \cap C$. Then $z_{n} \in C$ and $\left(x_{n}, z_{n}\right) \in F\left(t_{n}\right)$. So $\left\{\left(x_{n}, z_{n}\right)\right\}_{n \geq 1} \subseteq F\left(T_{0}\right)$, where $T_{0}=\left\{\left(t_{n}, t\right)\right\}_{n \geq 1}$. But since $F(\cdot)$ is u.s.c. and compact valued, it maps compact sets into compact sets (see Klein and Thompson [10], Theorem 7.4.2). So $F\left(T_{0}\right) \in P_{k}\left(R^{n} \times R^{n}\right)$. Hence $\left\{\left(x_{n}, z_{n}\right)\right\}_{n \geq 1}$ is relatively compact in $R^{n} \times R^{n}$ and so by passing to a subsequence if necessary, we may assume that $x_{n} \rightarrow x$ and $z_{n} \rightarrow z \Rightarrow(x, z) \in F(t) \Rightarrow z \in G(t, x)$ and $z \in C \Rightarrow z \in G(t, x) \cap C \Rightarrow(t, x) \in$ $G^{-}(C) \Rightarrow G(\cdot, \cdot)$ is u.s.c. as claimed. Applying Theorem 2.1 of $\mathrm{Ma}$ [11], we get $\hat{G}: R_{+} \times R^{n} \rightarrow P_{k c}\left(R^{n}\right)$ an u.s.c. extension of $G(\cdot, \cdot)$. Let $L$ be the graph of $\hat{G}(\cdot, \cdot)$. Since $\hat{G}(\cdot, \cdot)$ is u.s.c., $L$ is a closed subset of $R_{+} \times R^{n} \times R^{n}$. Let $q: R_{+} \times$ $R^{n} \times R^{n} \rightarrow \bar{R}=R \cup\{-\infty\}$ be defined by $q(t, x, z)=u(t, x, z)+\delta_{L}(t, x, z)$, where $\delta_{L}(\cdot, \cdot, \cdot)$ is the indicator function of the set $L$ (i.e. $\delta_{L}(t, x, z)=0$ if $(t, x, z) \in L$ and $-\infty$ otherwise). It is clear from this definition that $(t, x, z) \rightarrow q(t, x, z)$ is measurable, $(x, z) \rightarrow q(t, x, z)$ is upper semicontinuous and because of the convexity of $\hat{G}(t, x), z \rightarrow q(t, x, z)$ is concave. In terms of $q(\cdot, \cdot, \cdot)$, our variational problem $\left({ }^{*}\right)$ takes the following unconstrained form:

$$
\begin{aligned}
& \int_{0}^{\infty} q(t, x(t), \dot{x}(t)) d t \rightarrow \sup \\
& \text { over all } x: R_{+} \rightarrow R^{n} \text { absolutely continuous, } \\
& x(0)=x_{0} .
\end{aligned}
$$

Roughly speaking, what we did above is to impose an infinite penalty when the technological constraints are violated. 
Let $m$ be the value of our variational problem. Because of hypothesis $H_{\alpha}\left(x_{0}\right)$, $m>-\infty$. Let $S=\left\{\left(x_{n}, \dot{x}_{n}\right)\right\}_{n \geq 1}$ be a maximising sequence. From hypothesis $H(F)(2)$, for every $x(\cdot) \in S$ we have that if $\|x(t)\| \geq N(t)$ a.e., then $\langle x(t), \dot{x}(t)\rangle$ $\leq 0$ a.e.

$$
\begin{aligned}
& \Rightarrow \frac{1}{2} \frac{d}{d t}\langle x(t), x(t)\rangle=\frac{1}{2} \frac{d}{d t}\|x(t)\|^{2} \leq 0 \text { a.e. } \\
& \Rightarrow\|x(t)\|^{2} \leq\left\|x_{0}\right\|^{2} \\
& \Rightarrow\|x(t)\| \leq\left\|x_{0}\right\| .
\end{aligned}
$$

Thus finally, we can write that $\|x(t)\| \leq \max \left(N(t),\left\|x_{0}\right\|\right)=\hat{N}(t)$ a.e., and $\hat{N}(\cdot) \in L_{+}^{\infty}$. Also from hypothesis $H(F)(3)$, we have that $\|\dot{x}(t)\| \leq \varphi(t, \hat{N}(t))$ a.e. and $\varphi(\cdot, \hat{N}(\cdot)) \in L_{+}^{1}$. Now consider the set $W_{1} \subseteq C_{n}\left(R_{+}\right)$defined by

$$
\begin{gathered}
W_{1}=\left\{y(\cdot) \in C_{n}\left(R_{+}\right): y(t)=x_{0}+\int_{0}^{t} f(s) d s, t \in R_{+},\right. \\
\|f(s)\| \leq \varphi(s, \hat{N}(s)) \text { a.e. }\} .
\end{gathered}
$$

A straightforward application of the Arzela-Ascoli theorem tells us that $W_{1}$ is compact in $C_{n}\left(R_{+}\right)$with the topology of uniform convergence on compact sets in $R_{+}$(compact-open topology). Also let $W_{2} \subseteq L_{n}^{1}\left(R_{+}\right)$be defined by $W_{2}=\left\{f \in L_{n}^{1}\left(R_{+}\right):\|f(s)\| \leq \varphi(s, \hat{N}(s))\right.$ a.e. $\}$.

From the Dunford-Pettis compactness criterion we get that $W_{2}$ is weakly sequentially compact in $L_{n}^{1}\left(R_{+}\right)$. Now note that $S \subseteq W_{1} \times W_{2}$. So by passing to a subsequence if necessary, we may assume that $x_{n} \rightarrow x$ in $C_{n}\left(R_{+}\right)$and $\dot{x}_{n} \stackrel{w}{\rightarrow} z=\dot{x}$ in $L_{n}^{1}\left(R_{+}\right)$.

If $J(x, \dot{x})=\int_{0}^{\infty} q(t, x(t), \dot{x}(t)) d t$, without loss of generality we may assume that for all $n \geq 1, m-1 \leq J\left(x_{n}, \dot{x}_{n}\right) \leq m$. Since $m>-\infty$, we get that $q\left(t, x_{n}(t), \dot{x}_{n}(t)\right)>-\infty$ a.e. and so $q\left(t, x_{n}(t), \dot{x}_{n}(t)\right)=u\left(t, x_{n}(t), \dot{x}_{n}(t)\right), n \geq 1$. Because of hypothesis $H(u)(4)$, we can apply Theorem 2.1 of Balder [3], to get that

$$
m=\varlimsup \int_{0}^{\infty} u\left(t, x_{n}(t), \dot{x}_{n}(t)\right) d t \leq \int_{0}^{\infty} u(t, x(t), \dot{x}(t)) d t .
$$

Since $x(\cdot)$ is feasible, we have that $m=\int_{0}^{\infty} u(t, x(t), \dot{x}(t)) d t \Rightarrow x(\cdot)$ is the desired optimal path.

\section{Sensitivity analysis}

In this section we investigate the dependence of the optimal path of capital accumulation on the initial capital stock.

We shall need the following set of hypotheses:

$\underline{H(u)}_{1}: H(u)(1),(2)$ and (4) remain the same, while $H(u)(3)$ becomes $(x, z) \rightarrow$ $u(t, x, z)$. 
$\underline{H(F)_{1}}$ : The same as hypothesis $H(F)$, with the addition that $F(\cdot)$ is convex valued.

Hence the convexity of $G(t, x)$ is automatically satisfied. Let $K=F_{1}(0)$ $=\operatorname{proj}_{1} F(0)$. Then $K$ is a convex subset in $R^{n}$ and so it is well known that its relative interior rint $K$ is nonempty.

$\underline{H}_{\alpha}^{1}:$ For every $b \in \operatorname{rint} K$, hypothesis $H_{\alpha}(b)$ holds.

Then according to Theorem 1 , for every $b \in \operatorname{rint} K$, if the above hypotheses hold, problem $\left(^{*}\right)$ has solutions. Let $L: \operatorname{rint} K \rightarrow 2^{C_{n}\left(R_{+}\right) \times L_{n}^{1}\left(R_{+}\right)} \backslash\{\varnothing\}$ be the multifunction that, to each initial capital stock $b \in \operatorname{rint} K$, assigns the optimal "accumulation-investment paths" starting from $b$, that is,

$$
L(b)=\left\{(x(\cdot), \dot{x}(\cdot))=\text { optimal pair for problem }(*) \text {, with } x_{0}=b\right\} .
$$

THEOREM 2. If hypotheses $H(u)_{1}, H(F)_{1}$ and $H_{\alpha}^{1}$ hold, then $L(\cdot)$ is u.s.c. from rint $K$ into $C_{n}\left(R_{+}\right) \times L_{n}^{1}\left(R_{+}\right)_{w}$ and has nonempty, convex, compact values in this product space (recall that $L_{n}^{1}\left(R_{+}\right)_{w}$ denotes the space $L_{n}^{1}\left(R_{+}\right)$with the weak topology).

PROOF. Let $W_{1}^{\prime} \subseteq C_{n}\left(R_{+}\right)$be defined by

$$
\begin{aligned}
W_{1}^{\prime}=\left\{x(\cdot) \in C_{n}\left(R_{+}\right): x(t)=b+\int_{0}^{t} f(s) d s, t \in R_{+}, b \in K\right. & \\
& \|f(s)\| \leq \varphi(s, \hat{N}(s)) \text { a.e. }\} .
\end{aligned}
$$

Again by the Arzela-Ascoli theorem, since $K=F_{1}(0)$ is compact in $R^{n}$, we deduce that $W_{1}^{\prime}$ is compact for the topology of uniform convergence on compact subsets of $R_{+}$.

Also let $W_{2}$ be as in the proof of Theorem 1 . We have seen that $W_{2}$ is weakly sequentially compact in $L_{n}^{1}\left(R_{+}\right)$. Hence $W_{1}^{\prime} \times W_{2}$ is a sequentially compact subset of $C_{n}\left(R_{+}\right) \times L_{n}^{\mathbf{1}}\left(R_{+}\right)_{w}$. Observe that $L(\cdot)$ has closed values in $W_{1}^{\prime} \times W_{2}$. Thus in order to prove the theorem, it suffices to show that $G r L$ is closed in $W_{1}^{\prime} \times W_{2}$ with the relative $C_{n}\left(R_{+}\right) \times L_{n}^{1}\left(R_{+}\right)_{w}$-topology. Let $\left(b_{n}, x_{n}, \dot{x}_{n}\right) \rightarrow(b, x, z)$ in rint $K \times W_{1}^{\prime} \times W_{2}$, with $b \in \operatorname{rint} K$. It is clear that $\dot{x}=z$. Then if for each $n \geq 1, v\left(b_{n}\right)$ denotes the value of problem $\left(^{*}\right)$ for the initial capital stock $b_{n}$, we have $J\left(x_{n}, \dot{x}_{n}\right)=v\left(b_{n}\right)$. From Theorem 2.1 of Balder [3], we have that

$$
\varlimsup J\left(x_{n}, \dot{x}_{n}\right) \leq J(x, \dot{x}) \text {. }
$$

On the other hand, observe that $v(\cdot)$ is concave and finite on rint $K$. So it is continuous there (see for example Rockafellar [16]). Thus $v\left(b_{n}\right) \rightarrow v(b)$. Furthermore from Theorem 3.1 of [13], we have that

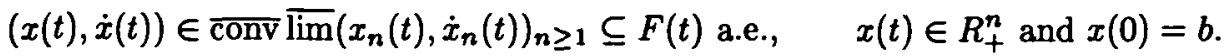


(Here $\overline{\lim }\left(x_{n}(t), \dot{x}_{n}(t)\right)_{n \geq 1}$ is the set of all subsequential limits of $\left\{\left(x_{n}(t)\right.\right.$, $\left.\left.\left.\dot{x}_{n}(t)\right)\right\}_{n \geq 1}\right)$.

Thus $x(\cdot)$ is a feasible capital accumulation path starting at $b$. Since $v(b) \leq$ $J(x, \dot{x})$, we conclude that $v(b)=J(x, \dot{x}) \Rightarrow(x, \dot{x}) \in G r L$. Therefore $\operatorname{GrL}$ is closed in $W_{1}^{\prime} \times W_{2}$ with the relative $C_{n}\left(R_{+}\right) \times L_{n}^{1}\left(R_{+}\right)_{w}$-topology. Hence $L(\cdot)$ is u.s.c. from rint $K$ into $C_{n}\left(R_{+}\right) \times L_{n}^{1}\left(R_{+}\right)_{w}$ and has nonempty, compact, convex values in the above product space.

REMARKS. (1) If the optimal path is unique (this is the case if for example $z \rightarrow u(t, x, z)$ is strictly concave), then Theorem 2 tells us that the single valued map $L(\cdot)$ is continuous from rint $K$ into $C_{n}\left(R_{+}\right) \times L_{n}^{1}\left(R_{+}\right)_{w}$.

(2) For each $t \geq 0$, the map $b \rightarrow L_{1}(b)(t)=\{x(t): x(\cdot)=$ optimal path $\}$ is u.s.c. from rint $K$ into $P_{k c} \in R^{n}$ ). In the case of uniqueness of the optimal path, this map is continuous.

\section{Acknowledgements}

The authors would like to express their gratitude to the referee and Prof. John Rickard for their many suggestions and remarks on how to improve the presentation of the material in this paper. The research was supported by N.S.F. Grant D.M.S.-8602313.

\section{References}

[1] K. Arrow and M. Kurz, Public investment, the rate of return and optimal fiscal policy (The Johns Hopkins Press, Baltimore, 1970).

[2] R. Ash, Real analysis and probability (Academic Press, New York, 1970).

[3] E. Balder, "Lower closure for orientor fields by lower semicontinuity of outer integral functionals", Annali di Mat. Pura ed Applicata 139 (1985) 349-360.

[4] L. M. Benveniste and J. Sheinkman, "Duality theory for dynamic optimization models in economics: The continuous time case", J. Econ. Theory 27 (1982) 1-19.

[5] W. Brock, "Sensitivity of optimal growth with respect to a change in target stocks", in Contributions to the Von-Neumann growth model (eds. G. Bruckmann and W. Weber) (Springer, Berlin 1971).

[6] D. Cass and K. Shell, "The structure and stability of competitive dynamical systems", J. Econ. Theory 12 (1976) 31-70.

[7] L. Cesari, "Closure, lower closure and seminormality theorems in optimal control", SIAM J. Control 9 (1971) 287-315.

[8] R. Gaines and J. Peterson, "The existence of optimal consumption policies in optimal economic growth models with nonconvex technologies", $J$. Econ. Theory 37 (1985) 76-98.

[9] A. Haurie, "Optimal control on an infinite time horizon: The turnpike approach", $J$. Math. Econ. 3 (1976) 81-102.

[10] E. Klein and A. Thompson, Theory of correspondences (Wiley, New York, 1984). 
[11] T. A. Ma, "Topological degree of set valued compact fields in locally convex spaces", Dissertationes Math. 92 (1972) 1-47.

[12] J. A. Mirrelees, "Optimum growth when technology is changing", Review of Economic Studies 34 (1967) 95-124.

[13] N. S. Papageorgiou, "Convergence theorems for Banach space valued integrable multifunctions", Intern. J. Math. and Math. Sci. 10 (1987) 433-442.

[14] F. P. Ramsey, "A mathematical theory of saving", The Economic Journal 38 (1928) 542559.

[15] R. T. Rockafellar, "Conjugate convex functions in optimal control and the calculus of variations", J. Math. Anal. Appl. 32 (1970) 174-222.

[16] R. T. Rockafellar, Convex Analysis (Princeton Univ. Press, Princeton, 1970).

[17] S. I. Takekuma, "A sensitivity analysis on optimal economic growth", J. Math. Econ. 7 (1980) 193-208. 